INTERDISZIPLINÄRE BEITRÄGE

ZUR SOZIALGESCHICHTE

122 Der Spitalfriedhof und das Bürgerspital zu Basel

Gerhard Hotz unter Mitarbeit

von Marina Zulauf-Semmler und

Verena Fiebig-Ebneter

132 Lesen in einem besonderen Archiv: der Mensch als Geschichtsquelle

Sandra Pichler 


\title{
LESEN IN EINEM BESONDEREN ARCHIV: DER MENSCH ALS GESCHICHTSQUELLE
}

\author{
1 DAS «ARCHIV MENSCH»
}

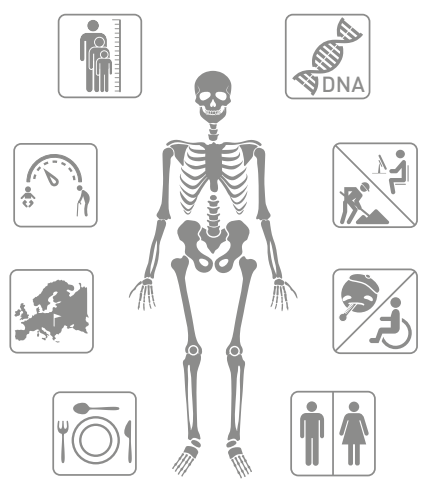

ABB. 1 Das menschliche Skelett beinhaltet zahlreiche Informationen zu Leben und Umwelt. Die Daten zu Sterbealter, Geschlecht, Körperhöhe, Arbeits- und Krankheitsbelastung, Ernährung, persönlichem Genom und geografischer Herkunft werden mit Hilfe vielfältiger Methoden erschlossen. Grafik: Matthias Coscoñas.
Der Mensch hinterlässt nicht nur Aufzeichnungen und Gegenstände aus vergangenen Zeiten, auch die körperlichen Überreste unserer Vorfahren bewahren detaillierte Informationen. Skelette, Leichenbrand oder Mumien sind ein Archiv, das Auskünfte geben kann über das Individuum und die Bevölkerung, aus der es stammt, über Ernährung und Erkrankungen, über Lebens- und Arbeitsbedingungen, persönliche Gewohnheiten und soziale Praktiken. Ebenso sind in ihnen Angaben zur natürlichen Umwelt und zum Klima gespeichert, in dem ein Mensch gelebt hat. ${ }^{1}$ (ABB. 1) Erschlossen wird dieses Archiv von der Archäoanthropologie. In enger Zusammenarbeit mit der Archäologie untersucht sie die Überreste ehemaliger menschlicher Bevölkerungen.

Zur Entschlüsselung der gespeicherten Informationen wird ein breites Spektrum von Methoden aus den Natur- wie den Geisteswissenschaften eingesetzt. So werden Erkenntnisse über die physiologischen (biologischen) Vorgänge im Laufe eines einzelnen Lebens gewonnen und - durch eine integrative multidisziplinäre Herangehensweise auch weitreichende sozialgeschichtliche Aussagen ermöglicht. Die klassischen osteologischen Verfahren sind seit langem etabliert und bedürfen daher keiner ausführlichen Darstellung. ${ }^{2}$ Im Folgenden sollen die neuesten naturwissenschaftlichen Methoden sowie deren sozialgeschichtlichen Implikationen im Vordergrund stehen.

\section{ARBEITSBELASTUNG UND KRANKHEIT}

Die Bedingungen, unter denen ein Mensch lebt und arbeitet, nehmen direkten Einfluss auf seine Gesundheit und sein Wohlbefinden. Am Skelett lässt sich z. B. die Schwere der körperliche Belastung an degenerativen Veränderungen und dem Vorkommen sogenannter «Enthesiopathien» abschätzen, d. h. an reaktiven Knochenstrukturen an den Ansatzstellen von Muskeln und Bändern sowie im Bereich von Gelenken. ${ }^{3}$ Sie liefern Hinweise auf spezifische Aktivitäten und Belastungsmuster und damit auf das Tätigkeitsspektrum von Männern und Frauen sowie die Einbindung von Kindern und Jugendlichen in die Aufgaben des Alltags.

Von allen Krankheiten, unter denen Menschen leiden, lässt sich nur ein geringer Teil am Knochen fassen. Am deutlichsten zeigen sich Verletzungen wie Knochenbrüche in Folge alltäglicher Unfälle oder zwischenmenschlicher Gewalt. Auch chirurgische Eingriffe wie die Schädeltrepanation (АВв.2) oder Amputationen sind fassbar. Infektionskrankheiten wie Tuberkulose oder Syphilis hinterlassen hingegen ihre Spuren am Skelett erst nach langer Krankheit. Parasiten, die ein ständiger Begleiter des Menschen waren und sind, tragen ebenfalls zu den unspezifischen Reaktionen des knöchernen Skeletts bei. Für die Diagnose pathologischer Veränderungen werden bildgebende (Röntgen, CT), aber auch biochemische Verfahren (z. B. ELISA) eingesetzt. ${ }^{4}$ In jüngster Zeit konnten Infektionskrankheiten auch mit Hilfe molekulargenetischer Verfahren nachgewiesen werden (s. u.). ${ }^{5}$ So sind z. B. aus Basel-Gasfabrik mehrere Spezies von Eingeweideparasiten in mikromorphologischen Dünnschliffen überliefert. (АВB. 3) Sie zeigen, dass die Parasiteneier im Boden der Siedlung verteilt waren und die Einwohner dadurch ständig der Gefahr einer Infektion ausgesetzt waren. ${ }^{6}$ Insgesamt aber weist die späteisenzeitliche Bevölkerung nur schwache Mangelanzeiger auf und scheint im Grossen und Ganzen recht gut ernährt gewesen zu sein. (АВв. 4, АВв. 5) Für die hohe Kindersterblichkeit in der latènezeitlichen Siedlung waren wohl Infektionskrankheiten die Ursache. ${ }^{7}$ Ohne die Einhaltung hygienischer Bedingungen und ohne eine verlässliche medizinische Versorgung sind sie auch heute für den Tod gerade von Kleinkindern verantwortlich. ${ }^{8}$ 
ABB. 2 Schädeltrepanation aus der latènezeitlichen Siedlung Basel-Gasfabrik. Die Operation liegt schon längere Zeit zurück und ist komplikationslos verheilt. Foto: Philippe Saurbeck.

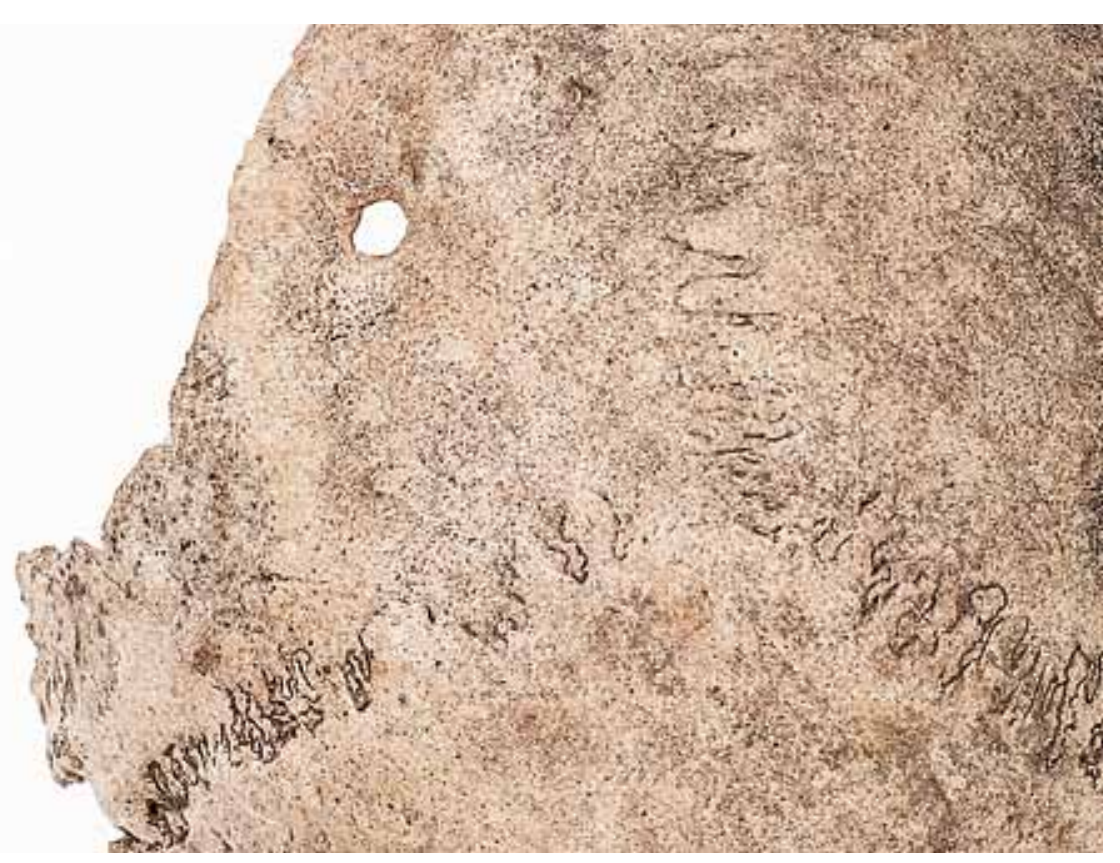

ABB. 3 Ei eines Spulwurms in einem mikromorphologischen Dünnschliff aus Basel-Gasfabrik. Foto: Christine Pümpin, IPNA.

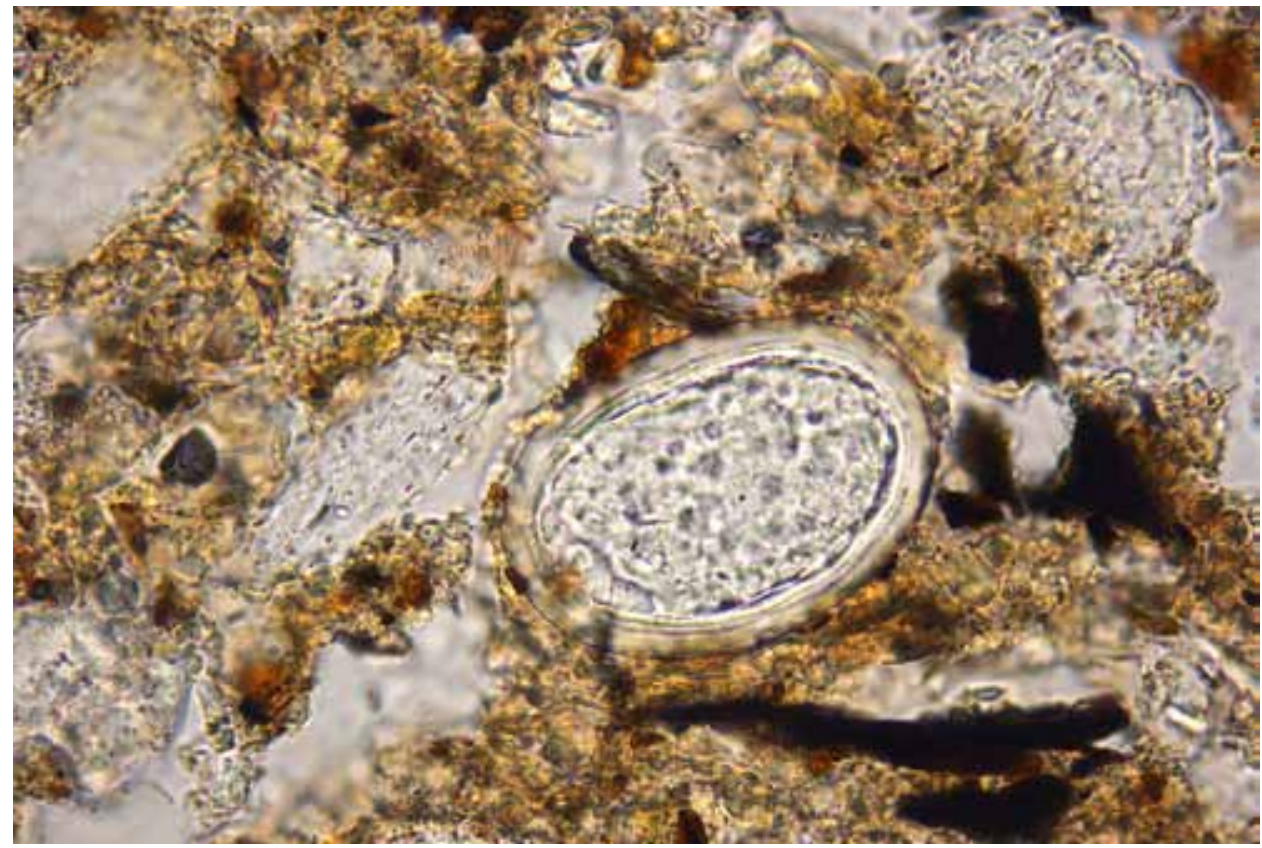

ABB. 4 Backenzahn eines etwa 4-jährigen Kindes mit einer Störung der Zahnschmelzbildung. Ursache ist eine Belastung durch Krankheit oder Mangel. Foto: Michael Wenk.

ABB. 5 Oberschenkelhals einer etwa 20-jährigen Frau mit Anzeichen von Vitamin C-Mangel. Foto: Philippe Saurbeck.

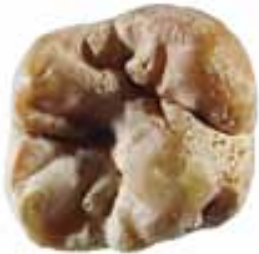

ABB. 4

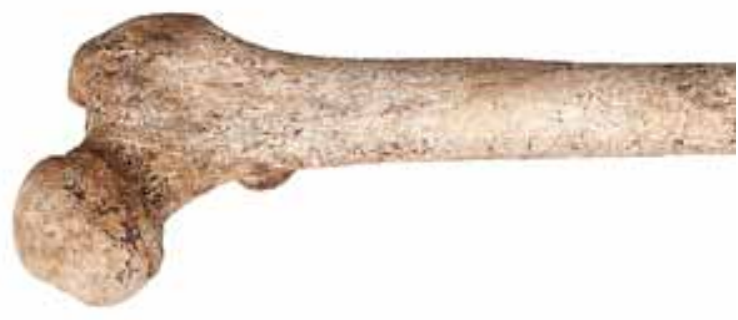

ABB. 5 


\section{SPEZIFISCHE EINBLICKE DURCH BIOARCHÄOMETRISCHE ANALYSEN}

Seit den 1980er Jahren gewinnen bioarchäometrische Analysen in der Archäoanthropologie zunehmend an Bedeutung. Die Labormethoden liefern Information zu Fragen der Ernährung, sozialer Praktiken wie Residenzmustern, Familienstrukturen oder der Länge von Stillperioden, spezifischer Krankheitserreger und der Mobilität und Migration. ${ }^{9}$ Sie eröffnen damit ein Fenster in die Vergangenheit, das mit grossem Detailreichtum Aspekte des täglichen Lebens erhellt. Der Erfolg der Analysen ist von der Güte des Probenmaterials abhängig. Die Entnahme der Proben bedarf daher gezielter Beprobungsstrategien und optimaler Rahmenbedingungen bei der Ausgrabung..$^{10}$ Die Aussagemöglichkeiten der beiden in der Archäoanthropologie wichtigsten Verfahren, der Analyse von stabilen Isotopen und der molekularbiologischen Untersuchungen, sollen im Folgenden kurz dargestellt werden.

\subsection{DIE ANALYSE STABILER ISOTOPE}

Isotope sind unterschiedliche Arten von Atomen eines Elementes, die sich in der Anzahl ihrer Neutronen unterscheiden. Damit ist die Ordnungszahl der Atome gleich, ihre Masse unterscheidet sich jedoch geringfügig. Diese Gewichtsunterschiede können mit Hilfe eines Massenspektrometers gemessen und die Isotope damit identifiziert werden. Über Stoffwechselvorgänge werden die chemischen Elemente mit ihren spezifischen Isotopenkonzentrationen in die Gewebe von Menschen, Tieren und Pflanzen eingebaut. Dies bildet die Basis für die Nutzung von stabilen Isotopen für archäologische und archäoanthropologische Fragestellungen. ${ }^{11}$

\subsubsection{ERNÄHRUNGSREKONSTRUKTION}

Das Verhältnis der stabilen Isotope Kohlenstoff ${ }^{13} \mathrm{C}$ und Stickstoff ${ }^{15} \mathrm{~N}$ im Knochenkollagen liefert Informationen zum Typ der überwiegend konsumierten Nahrungsmittel. Sie zeigen einerseits den Anteil pflanzlicher und tierischen Proteinquellen in der täglichen Ernährung und können andererseits spezifische Nahrungsmittel identifizieren. Dies sind z. B. die sogenannten $\mathrm{C}_{4}$-Pflanzen (z. B. Hirse), die über eine andersartige Photosynthese verfügen als die meisten unserer heimischen $\mathrm{C}_{3}$-Getreidearten wie der Weizen. Auch Nahrungsmittel marinen Ursprungs fallen durch ihre abweichenden Isotopenprofile auf. Die Einbringung zusätzlicher Nährstoffe in den Boden (Düngung), die dann von Pflanzen (Produzenten) und Tieren oder Menschen (Konsumenten) aufgenommen werden, ist ebenfalls in den Isotopenspektren erkennbar. Für die Analysen eignen sich besonders Rippen, da sie eine schnelle Umbaurate haben und so die Ernährung der letzten Lebensjahre eines Individuums widerspiegeln. (АВB. 6, АВB. 7) Aus den Daten der Analysen von $\mathrm{C}$ und $\mathrm{N}$ ergibt sich eine Vielzahl von wirtschafts- und sozialgeschichtlichen Einblicken. So wird z. B. der Anteil von tierischen Proteinen, für deren Erzeugung ein höherer Aufwand nötig ist als bei Pflanzen, als Indikator für gesellschaftliche Verhältnisse herangezogen. Für Basel-Gasfabrik lassen die Analysewerte eine recht einheitliche Ernährung der Menschen erkennen. (ABB. 8) Die Variation hin zur rechten Seite des Diagramms zeigt bei Mensch und Huhn den Konsum von Hirse an, die als $\mathrm{C}_{4}$-Pflanze unter den botanischen Resten nachgewiesen ist. Kleinkinder hingegen nehmen durch die Muttermilch ausschliesslich tierische Proteine zu sich. ${ }^{12}$ Sobald begonnen wird, den Kindern Getreidebrei o. ä. zuzufüttern, verändert die pflanzliche Komponente die Isotopenwerte. So kann auf das Abstillalter der Kinder geschlossen werden. 
ABB. 7 Eignung von Skelettelementen für

Analysen von stabilen Isotopen und

aDNA-Untersuchungen (modifiziert nach

Alt et al. 2014). Grafik: Matthias Coscoñas.

Zahnproben (aDNA)

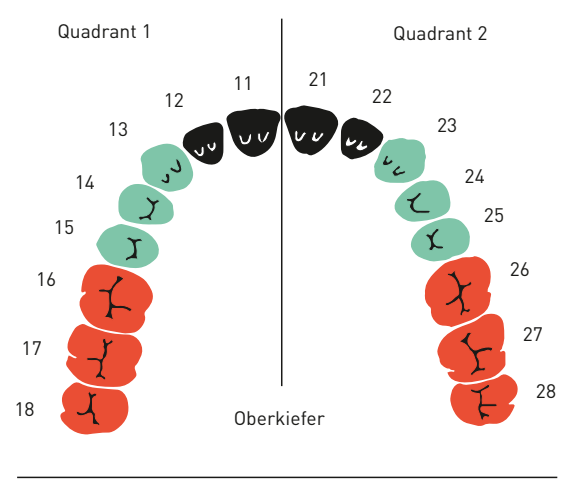

48

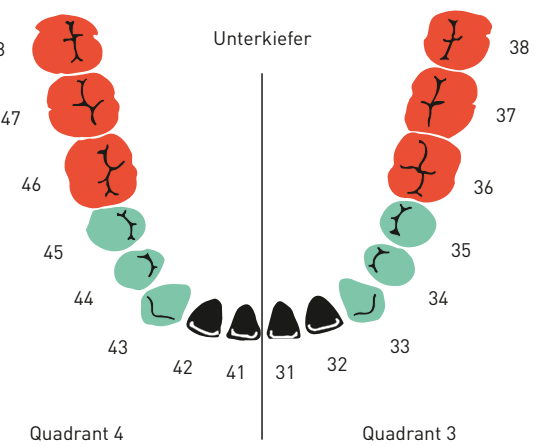

Knochenproben (aDNA)

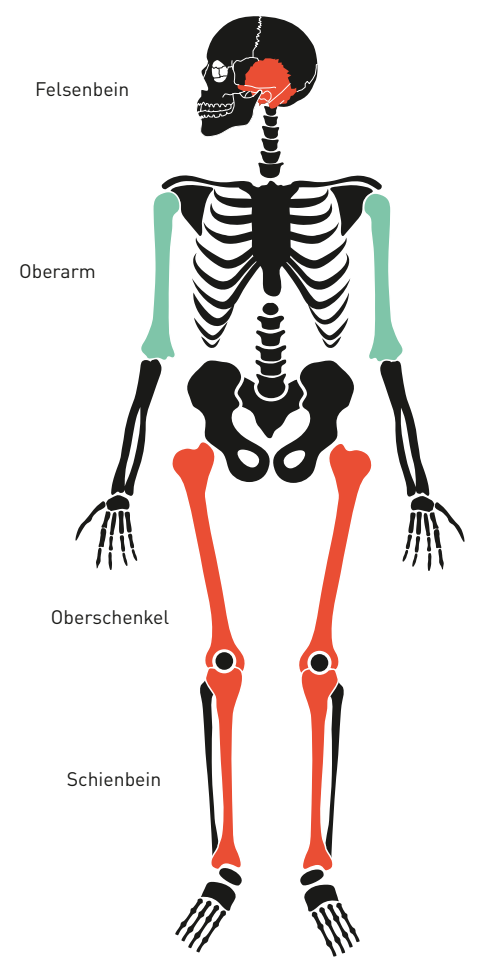

而

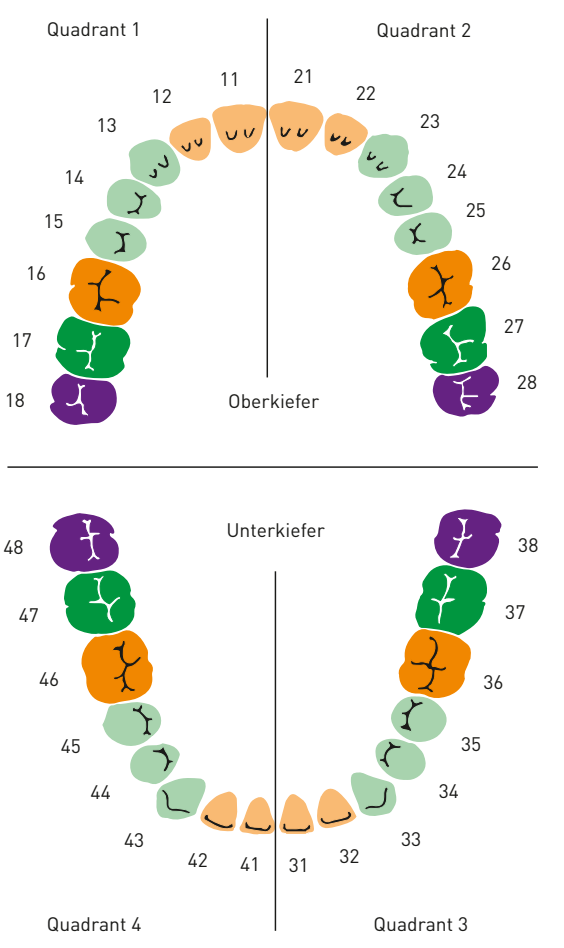

Bildung

der Zahnkrone

früh

(Geburt bis $3 / 5$ Jahre)

mittel

(2 bis 7 Jahre)

spät
(7 bis

Besonders geeignet

Alternativen

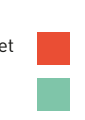$$
\text { . }
$$ 
ABB. 8 Ernährungrekonstruktion der Bewohner von Basel-Gasfabrik mit Hilfe von stabilen Kohlenstoff- und Stickstoffisotopen (modifiziert nach Knipper et al. 2015). Grafik: Matthias Coscoñas.

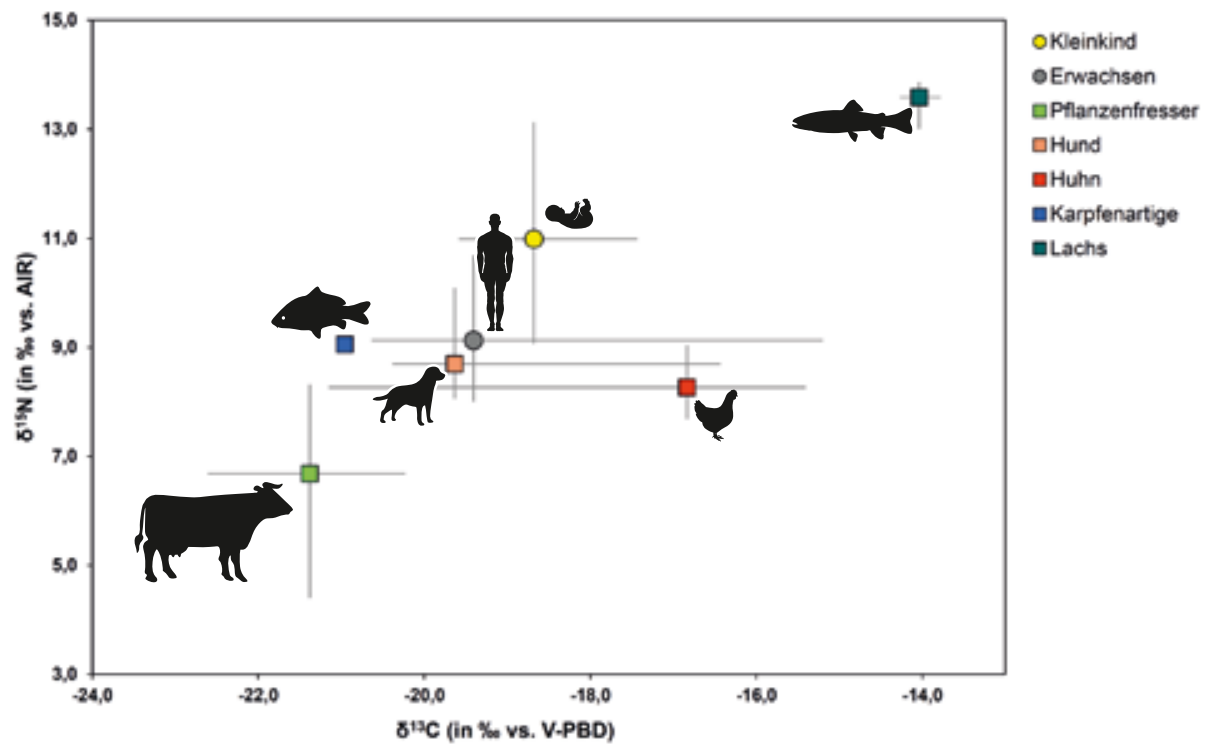

\subsubsection{MOBILITÄT}

Die Mobilität eines Menschen wird mit Hilfe der Verhältnisse von Strontium ${ }^{87} \mathrm{Sr} /{ }^{86} \mathrm{Sr}$ und Sauerstoff ${ }^{18} \mathrm{O}$ im Zahnschmelz erschlossen. Strontium gelangt über die Pflanzen aus dem Boden in die Nahrungskette. Unterschiedliche Gesteine und Böden weisen ganz typische Strontiumsignaturen auf, so dass jede Region durch bestimmte Werte gekennzeichnet ist. Sauerstoff wird hauptsächlich über das Trinkwasser aufgenommen und seine Isotopenverhältnisse sind geprägt durch die Niederschläge und Klimaverhältnisse einer Region; so lassen sich z. B. Hochgebirge und Flussebenen unterscheiden. Die Zahnkronen, die für die Analysen von $\mathrm{Sr}$ und $\mathrm{O}$ beprobt werden, entwickeln sich im Laufe von Kindheit und Jugend und weisen die Isotopensignaturen derjenigen Region auf, in der ein Mensch in diesem Zeitraum gelebt hat. Deshalb werden für die Beprobung möglichst die zuerst und zuletzt gebildeten Zähne gewählt. (ABB. 7)

Auch für Sr- und 0-Analysen ist die Probenentnahme unproblematisch, da keine direkte Kontaminationsgefahr besteht oder besonderen Lagerungsvorschriften zu beachten sind. Strontium- und Sauerstoffanalysen erlauben ein breites Spektrum von Aussagen. Diese betreffen zum einen die Mobilität. Anhand der Isotopenwerte können lokale und ortsfremde Individuen unterschieden werden. Im Idealfall lässt sich sogar die Herkunftsregion von Migranten identifizieren. Werden unterschiedliche Werte für verschiedene Zähne gemessen, so erhält man Hinweise auf einen Ortswechsel des Individuums. Da die Zeiträume bekannt sind, in denen die Zahnkronen wachsen, lässt sich sogar das Lebensalter bestimmen, in dem der Ortswechsel stattfand. Die Isotopenwerte spiegeln auch weitere soziale Praktiken, etwa die der Matri- oder Patrilokalität von Gemeinschaften oder der Grösse der Heiratskreise: die ortstypischen Signaturen zeigen, woher die Heiratspartner kamen, und ob z. B. immer Frauen aus einer bestimmten Region eingeheiratet haben. Über die Einbeziehung von Tieren und Pflanzen können zudem wirtschaftsarchäologische Fragen untersucht werden. So wurde in Basel-Gasfabrik festgestellt, dass ein Teil der Schlachttiere aus dem weiteren Umland «importiert» wurde. ${ }^{13}$ Wieder andere Untersuchungen bieten Hinweise auf saisonale Weidewirtschaft in Gebirgsgegenden, die sogenannte Transhumanz. ${ }^{14}$

Seit wenigen Jahren werden für Herkunftsuntersuchungen auch andere Isotopensysteme auf ihre Aussagekraft hin untersucht, so z. B. die leichten Elemente Wasserstoff $(\mathrm{H})$ und Schwefel (S) oder das schwere Element Blei (Pb). Die Methoden sind hier allesamt noch in der Entwicklung und zukünftige Untersuchungen werden zeigen, ob dadurch qualitativ bessere Ergebnisse zu erzielen sind. 


\subsection{MOLEKULARGENETISCHE ANALYSEN}

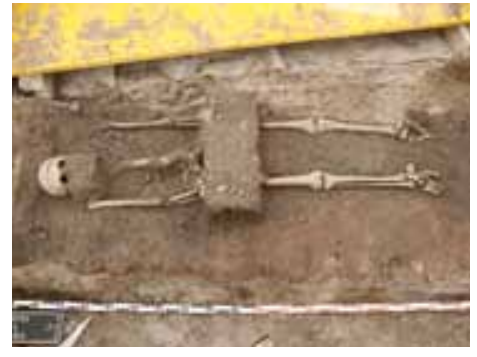

ABB. 9 Intakte Erdabdeckung von Kieferund Oberschenkelbereich zur Vermeidung von Kontaminationen bei der Probenentnahme für molekulargenetische Analysen. Foto: Benedikt Wyss.

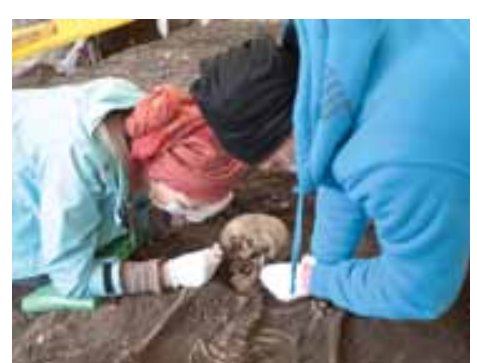

ABB. 10 Probenentnahme für aDNA-Analysen auf der Lehrgrabung Spitalfriedhof St. Johann. Foto: Benedikt Wyss.
Das Erbgut ist in den Zellen von Lebewesen in Form eines langen Kettenmoleküls, der sogenannten Desoxyribonukleinsäure (DNA) gespeichert. Nach dem Tod eines Individuums wird die DNA allmählich abgebaut, die langen Ketten zerfallen in immer kleinere Bruchstücke. Mit Hilfe der Polymerase-Kettenreaktion wurde es in den 1980er Jahren möglich, kurze Abschnitte von a(lter) DNA zu analysieren. Inzwischen kann mit einem als «Next Generation Sequencing» bezeichneten Verfahren selbst die gesamte Erbinformation eines Individuums, das sogenannte Genom, entschlüsselt werden. ${ }^{15}$

Für konventionelle aDNA-Analysen sind Zähne, speziell Molaren besonders geeignet, da das Dentin, an dem die Untersuchungen vorgenommen werden, durch die Schmelzüberdeckung geschützt ist. Beim «Next Generation Sequencing» haben sich darüber hinaus Proben aus dem Felsenbein bewährt, dessen dichte kompakte Knochensubstanz in der Regel ebenfalls gut erhalten ist. Eine grosse Herausforderung bei aDNAAnalysen stellt die Kontaminationsvermeidung dar. Hautschuppen, Schweisstropfen o. ä. von lebenden Menschen, die mit den alten Überresten in Kontakt kommen, enthalten grosse Mengen Genmaterial und können verhindern, dass die endogene aDNA eindeutig zu identifizieren ist. Damit werden Ergebnisse verfälscht und sind unbrauchbar. Um dies zu vermeiden, sind bei den Ausgrabungsarbeiten und im Labor umfangreiche Vorsichtsmassnahmen und Schutzkleidung nötig. (ABB. 9, АВB. 10)

Molekulargenetische Analysen bringen spezifische Erkenntnisse zu einer grossen Zahl von Forschungsthemen und eröffnen damit neue Perspektiven auf den Menschen wie auch seine ausgestorbenen Vorfahren. ${ }^{16}$ Entwicklungs- und umweltabhängige Parameter wie das individuelle Sterbealter, die Körperhöhe, der generelle Gesundheitszustand, sowie habituelle und artifizielle Veränderungen bleiben dagegen weiterhin den «klassischen» Untersuchungsmethoden vorbehalten.

\subsubsection{GENETISCHE DATEN ZUM INDIVIDUUM}

Zu den ältesten molekulargenetischen Verfahren, die in der Archäoanthropologie Anwendung finden, gehört die Geschlechtsbestimmung. ${ }^{17}$ Sie ist, bei entsprechender Erhaltung, auch bei Subadulten möglich und leistet somit einen wichtigen Beitrag zu demografischen Fragestellungen. Auch die Speziesbestimmung bzw. die Unterscheidung von Tier- und Menschenknochen kam bei stark fragmentierten Skelettresten bereits früh zum Einsatz. Mit verbesserten Methoden werden in den letzten Jahren auch Detailinformationen greifbar: von der Haar- und Hautfarbe bis hin zu physiologischen Parametern wie der Fähigkeit, Milch oder Alkohol zu verdauen. ${ }^{18}$ Die Verwandtschaftsanalyse von Mitgliedern menschlicher Bestattungsgemeinschaften war ebenfalls bereits früh Gegenstand der Forschung. Mit ihrer Hilfe ist es möglich, genetisch verwandte Individuen oder auch historische Persönlichkeiten zu identifizieren und damit einen Einblick zu erhalten in die sozialen Strukturen ehemaliger Bevölkerungen. ${ }^{19}$ 


\subsubsection{GENETISCHE DATEN ZU POPULATIONEN}

Durch die Verbreitung und Häufigkeit sogenannter Haplogruppen, d. h. von Individuen, die über die mütterliche (mtDNA) oder väterliche (Y-DNA) Linie gemeinsame Vorfahren haben, lassen sich die Populationen eines geographischen Grossraumes, etwa Europas, charakterisieren. Tiefgreifende Veränderungen in deren genetischer Zusammensetzung sind Anzeiger eines Bevölkerungswechsels bzw. von Migrationsbewegungen. Derartige Migrationsereignisse sind z. B. aus dem Neolithikum bekannt, als die ersten Bauern aus dem Donauraum nach Mitteleuropa vordrangen und die autochthone Bevölkerung weitgehend verdrängten. ${ }^{20}$ Eine weitere Migrationswelle brachte 3 Jahrtausende später Menschen, die von den Steppenvölkern Westasiens abstammten. ${ }^{21}$

Ebenfalls auf dem Populationsniveau lassen sich molekulargenetisch Adaptationsund Selektionsprozesse fassen, wie z. B. die Herausbildung der roten Haarfarbe in Europa. ${ }^{22}$ Von grossem Interesse ist auch der Ursprung von Zivilisationskrankheiten wie Diabetes oder der Nachweis von Krankheiten wie der Pest. ${ }^{23}$ Erst kürzlich ist es gelungen, das Bakterium Heliobacter pylori in den Eingeweiden der Eismumie Ötzi nachzuweisen, das Geschwüre im Magen und im Zwölffingerdarm verursacht. ${ }^{24}$

\subsubsection{MIKROBIOMANALYSEN}

Der jüngste Zweig der molekulargenetischen Forschung hat das sog. Mikrobiom zum Gegenstand, d. h. nicht den Menschen selbst, sondern die zahlreichen Organismen, die wir beheimaten. So konnte nachgewiesen werden, dass die Veränderungen in der menschlichen Ernährung über die Jahrtausende zu einer deutlichen Verarmung unserer Darmflora geführt hat, die eine wichtige Rolle in der Immunabwehr spielt. ${ }^{25}$ Auch in der Mundflora spiegeln sich die massiven Umwälzungen unserer Lebensweise mit der Einführung einer produzierenden Wirtschaftsweise im Neolithikum und noch deutlicher mit der Industrialisierung im 19. Jahrhundert. In der Folge haben wir heute mehr Bakterienstämme in der Mundhöhle, die Karies und Parodontitis verursachen. ${ }^{26}$

\section{FAZIT}

Das «Archiv Mensch» erlaubt direkte Einblicke in die «life history» Einzelner wie auch in die Bedingungen, unter denen Menschen zu verschiedenen Zeiten lebten. Gerade die letzten drei Jahrzehnte haben dabei grundlegende Neuerungen gebracht: die wachsende Bedeutung von Labormethoden, eine verstärkte Einbeziehung statistischer Verfahren, und die Entwicklung disziplinübergreifender Fragestellungen und Aussagemöglichkeiten. In enger Zusammenarbeit von Natur- und Geisteswissenschaften wird es möglich nachzuvollziehen, wie sich Menschen über die ihnen inhärente Kombination aus Natur und Kultur umweltinduzierten wie sozialen Herausforderungen stellten und damit ein detailreiches Bild der Vergangenheit zu zeichnen. 


\section{ANMERKUNGEN}

1 Grupe et al. 2015.

2 Grupe et al. 2015.

3 Meyer et al. 2011.

4 Böni 1996.

5 Cooper et al. 2016.

6 Pichler et al. 2014.

7 Rissanen et al. 2013.

8 Black et al. 2010.

9 Grupe et al. 2015.

10 Alt et al. 2014.

11 Grupe et al. 2015.

12 Knipper et al. 2015.

13 Knipper et al. 2016.

14 Doppler et al. 2015.

15 Grupe et al. 2015.

16 Grupe et al. 2015.

17 Grupe et al. 2015.

18 Itan et al. 2009.

19 Haak et al. 2008;

Haas et al. 2014.

20 Haak et al. 2010.

21 Haak et al. 2015.

22 Lalueza-Fox et al. 2007.

23 Rasmusen et al. 2015.

24 Maixner et al. 2016.

25 Warinner, Lewis 2015.

26 Adler et al. 2013.

\section{Literatur}

C. J. Adler, K. Dobney, L. S. Weyrich, J. Kaidonis, A. W. Walker, W. Haak, C. J. A. Bradshaw, G. Townsend, A. Sottysiak, K. W. Alt, J. Parkhill, A. Cooper: Sequencing ancient calcified dental plaque shows changes in oral microbiota with dietary shifts of the Neolithic and Industrial revolutions in: Nature Genetics 45 (2013), 450-455.

K.W. Alt, G. Brandt, C. Knipper, C. Lehn: Empfehlungen für die Probenentnahme in der forensischen Anthropologie, in: Rechtsmedizin 24 (2014), 179-185.

R. E. Black, S. Cousens, H. L. Johnson, J. E. Lawn, I. Rudan, D. G. Bassani, P. Jha, H. Campbell, C. Fischer Walker, R. Cibulskis, T. Eisele, L. Liu, C. Mathers: Global, regional, and national causes of child mortality in 2008: a systematic analysis, in: The Lancet 375 (2010), 1969-1987.

T. Böni: Paläopathologie: eine Wissenschaft im Dienste der Archäologie, Anthropologie und Medizingeschichte, in: AS 19 (1996), 174-179.
C. Cooper, R. Fellner, O. Heubi, F. Maixner, A. Zink, S. Lösch: Tuberculosis in early medieval Switzerland - osteological and molecular evidence, in: Swiss medical weekly 146 (2016), w14269.

T. Doppler, C. Gerling, J. Schibler: The importance of the hinterland: Multi-isotope analysis on animals from Neolithic lakeshore settlements in the Alpine foreland, in: Past 80 (2015), 4-5.

G. Grupe, M. Harbeck, G. C. McGlynn: Prähistorische Anthropologie, Berlin 2015.

W. Haak, G. Brandt, H. N. de Jong, C. Meyer, R. Ganslmeier, V. Heyd, C. Hawkesworth, A. Pike, H. Meller, K. W. Alt: Ancient DNA, Strontium isotopes, and osteological analyses shed light on social and kinship organization of the Later Stone Age, in: Proceedings of the National Academy of Science 105 (2008), 18226-18231.

W. Haak, O. Balanovsky, J. J. Sanchez, S. Koshel, V. Zaporozhchenko, C. J. Adler, C. S. I. Der Sarkissian, G. Brandt, C. Schwarz, N. Nicklisch, V. Dresely, B. Fritsch, E. Balanovska, R. Villems, H. Meller, K. W. Alt, A. Cooper (the Genographic Consortium): Ancient DNA from European Early Neolithic Farmers Reveals Their Near Eastern Affinities, in: PLoS Biol 8 (2010), e1000536.

W. Haak, I. Lazaridis, N. Patterson, N. Rohland, S. Mallick, B. Llamas, G. Brandt, S. Nordenfelt, E. Harney, K. Stewardson, Q. Fu, A. Mittnik, E. Banffy, C. Economou, M. Francken, S. Friederich, R. G. Pena, F. Hallgren, V. Khartanovich,

A. Khokhlov, M. Kunst, P. Kuznetsov, H. Meller,

O. Mochalov, V. Moiseyev, N. Nicklisch, S. L. Pichler, R. Risch, M. A. Rojo Guerra, C. Roth, A. SzecsenyiNagy, J. Wahl, M. Meyer, J. Krause, D. Brown, D. Anthony, A. Cooper, K. W. Alt, D. Reich: Massive migration from the steppe was a source for Indo-European languages in Europe, in: Nature 522 (2015), 207-211.

C. Haas, N. Shved, F. J. Rühli, C. Papageorgopoulou, M. Krawczak, J. Purps, S. Willuweit: Molekulargenetische Abstammungsanalyse am mutmasslichen Skelett des Bündner Freiheitshelden Jörg Jenatsch, in: M. Janosa (Hg.): Unter die Orgl begraben: Das Grab des Jörg Jenatsch in der Kathedrale zu Chur, Chur 2014, 111-120.

Y. Itan, A. Powell, M. A. Beaumont, J. Burger, M. G. Thomas, 2009. The Origins of Lactase Persistence in Europe, in: PLoS Computational Biology 5 (2009), e1000491.

C. Knipper, S. Pichler, D. Brönnimann, K. W. Alt: Über den Tellerrand geschaut: High-Tech lässt alte Knochen sprechen, in: AS 38.2 (2015), 60-61.

C. Knipper, S. L. Pichler, H. Rissanen, B. Stopp, M. Kühn, N. Spichtig, B. Röder, J. Schibler, G. Lassau, K. W. Alt: What is on the menu in a Celtic town? Iron Age diet reconstructed at Basel-Gasfabrik, Switzerland, in: Archaeological and Anthropological Sciences (2016) DOI 10.1007/ s12520-016-0362-8.
C. Lalueza-Fox, H. Römpler, D. Caramelli, C. Stäubert, G. Catalano, D. Hughes, N. Rohland, E. Pilli, L. Longo, S. Condemi, M. de la Rasilla, J. Fortea, A. Rosas, M. Stoneking, T. Schöneberg, J. Bertranpetit, M. Hofreiter: A Melanocortin 1 Receptor Allele Suggests Varying Pigmentation among Neanderthals, in: Science 318 (2007), 1453-1455.

F. Maixner, B. Krause-Kyora, D. Turaev, A. Herbig, M. R. Hoopmann, J. L. Hallows, U. Kusebauch, E. E. Vigl, P. Malfertheiner, F. Megraud, N. O'Sullivan, G. Cipollini, V. Coia, M. Samadelli, L. Engstrand, B. Linz, R. L. Moritz, R. Grimm, J. Krause, A. Nebel, Y. Moodley, T. Rattei, A. Zink: The 5300-year-old Helicobacter pylori genome of the Iceman, in: Science 351 (2016), 162-165.

C. Meyer, N. Nicklisch, P. Held, B. Fritsch, K. W. Alt: Tracing patterns of activity in the human skeleton: An overview of methods, problems, and limits of interpretation, in: HOMO - Journal of Comparative Human Biology 62 (2011), 202-217.

S. L. Pichler, C. Pümpin, D. Brönnimann, P. Rentzel: Life in the Proto-Urban Style: The identification of parasite eggs in micromorphological thin sections from the Swiss Basel-Gasfabrik late Iron Age settlement, in: Journal of Archaeological Science 43 (2014), 55-65.

S. L. Pichler, G. Lassau, K. W. Alt, B. Röder, J. Schibler: Über die Toten zu den Lebenden. Interdisziplinäre Synthese, Materialhefte zur Archäologie in Basel 25, (Beiträge zu BaselGasfabrik), in Vorbereitung.

S. Rasmussen, M. E. Allentoft, K. Nielsen, L. Orlando, M. Sikora, K.-G. Sjögren, A. G. Pedersen, M. Schubert, A. Van Dam, C. M. O. Kapel, H. B. Nielsen, S. Brunak, P. Avetisyan, A. Epimakhov, M. V. Khalyapin, A. Gnuni, A. Kriiska, I. Lasak, M. Metspalu, V. Moiseyev, A. Gromov, D. Pokutta, L. Saag, L. Varul, L. Yepiskoposyan, T. SicheritzPontén, R. A. Foley, M. M. Lahr, R. Nielsen, K. Kristiansen, E. Willerslev: Early Divergent Strains of Yersinia pestis in Eurasia 5000 Years Ago, in: Cell 163 (2015), 571-582.

H. Rissanen, S. L. Pichler, N. Spichtig, K. W. Alt, D. Brönnimann, C. Knipper, M. Kühn, P. Rentzel, B. Röder, J. Schibler, B. Stopp, W. Vach, O. Warnberg, Guido Lassau: «Wenn Kinder sterben ...» Säuglinge und Kleinkinder aus dem latènezeitlichen Fundplatz Basel-Gasfabrik, in: S. Wefers, J. E. Fries, J. Fries-Knoblach, C. Later, U. Rambuschek, P. Trebsche, J. Wiethold (Hg.): Eisenzeit und Geschlechterforschung. Bilder - Räume - Rollen. Beiträge zur gemeinsamen Sitzung der AG Eisenzeit und der AG Geschlechterforschung während des 7. Deutschen Archäologiekongresses in Bremen 2011, Langenweissbach 2013, 127-142.

C. Warinner, C. M. Lewis: Microbiome and Health in Past and Present Human Populations, in: American Anthropologist 117 (2015), 740-741. 


\section{Allgemeine Abkürzungen}

ABBS Archäologische Bodenforschung des Kantons BaselStadt

AG Kanton Aargau

AUE Amt für Umwelt und Energie

BS Kanton Basel-Stadt

BVD Bau- und Verkehrsdepartement

bz Basellandschaftliche Zeitung

CT Computertomografie

DST Dienststelle

FK Fundkomplex

HMB Historisches Museum Basel

IPNA Institut für Integrative Prähistorische und Naturwissenschaftliche Archäologie (Universität Basel)

IWB Industrielle Werke Basel

PD Präsidialdepartement

StAAG Staatsarchiv Aargau

StABS Staatsarchiv Basel-Stadt

SNF Schweizerischer Nationalfond

SFR Schweizer Radio und Fernsehen

UMIS Umbau und Instandsetzung des Spiegelhofes

VS Kanton Wallis

\section{Literatur Abkürzungen}

AS Archäologie Schweiz

BZ Basler Zeitschrift für Geschichte und Altertumskunde

JASc Journal of Archaeological Science

JbAB Jahresbericht der Archäologischen Bodenforschung Basel-Stadt

JbAS Jahrbuch Archäologie Schweiz

KDM BS Die Kunstdenkmäler der Schweiz bzw. des Kantons Basel-Stadt

\section{Impressum}

Herausgeberin:

Archäologische Bodenforschung des Kantons Basel-Stadt Basel 2016

Redaktion: Andreas Niederhäuser

Bildredaktion: Philippe Saurbeck

Konzept und Gestaltung: New Identity Ltd., Basel

Lithografie und Druck: Steudler Press AG, Basel
Verlag und Bestelladresse:

Archäologische Bodenforschung des Kantons Basel-Stadt

Petersgraben 11

$\mathrm{CH}-4051$ Basel

Schweiz

E-Mail: arch.bodenforschungabs.ch

www.archaeologie.bs.ch

(c) 2016 Archäologische Bodenforschung des Kantons Basel-Stadt

Auflage: 800 Exemplare

ISBN 978-3-905098-62-4

ISSN 1424-4535

(c) Kartengrundlagen-Quelle: Geodaten Kanton Basel-Stadt. 